\title{
Utility of digital rectal examination in a population with prostate cancer treated with active surveillance
}

Jaime O. Herrera-Caceres, MD ${ }^{1}$; Marian S. Wettstein, MD ${ }^{1}$; Hanan Goldberg, MD ${ }^{1}$; Ants Toi, $\mathrm{MD}^{2}$; Thenappan Chandrasekar, $\mathrm{MD}^{3}$; Dixon T.S. Woon, MD ${ }^{1}$; Ardalan E. Ahmad, MD ${ }^{1}$; Noelia Sanmamed-Salgado, $\mathrm{MD}^{4}$; Omar Alhunaidi, MD ${ }^{1}$; Khaled Ajib, $\mathrm{MD}^{1}$; Gregory Nason, MD ${ }^{1}$; Guan Hee Tan, MD ${ }^{1}$; Neil Fleshner, MD, MPH ${ }^{1}$; Laurence Klotz, $\mathrm{MD}^{5}$

${ }^{1}$ Division of Urology, Department of Surgical Oncology, University of Toronto and University Health Network, Toronto, ON, Canada; ${ }^{2}$ Department of Medical Imaging, Princess Margaret Cancer Centre, University of Toronto, Toronto, ON, Canada; ${ }^{3}$ Department of Urology, Sidney Kimmel Cancer Center, Thomas Jefferson University, Philadelphia, PA, United States; ${ }^{4}$ Department of Radiation Oncology, University of Toronto and University Health Network, Toronto, ON, Canada; ${ }^{5}$ Division of Urology, Department of Surgery, Sunnybrook Health Sciences Centre, Toronto, ON, Canada

Cite as: Can Urol Assoc J 2020 March 30; Epub ahead of print. http://dx.doi.org/10.5489/cuaj.6341

Published online March 30, 2020

$* * *$

\section{Abstract}

Introduction: Digital rectal examination (DRE) is part of the clinical evaluation of men on active surveillance (AS). The purpose of the present study is to analyze the value of DRE as a predictor of upgrading in a population of men with prostate cancer (PC) treated with AS. Methods: We used the prostate biopsy (PBx) database from an academic center, including PBx from 2006-2018, and identified 2029 confirmatory biopsies (CxPBx) of men treated with AS, of which 726 men had both diagnostic (initial) and CxPBx information available. We did a descriptive analysis and evaluated sensitivity, specificity, and predictive values of DRE for the detection of clinically significant PC (csPC). Multivariable regression analysis was done to identify predictors of csPC. The primary outcome was to evaluate DRE as a predictor of the presence of $\operatorname{cs} \mathrm{PC}$ at $\mathrm{CxPBx}$.

Results: Among the 2029 patients with a CxPBx, 75\% had PC, and of these, 30.3\% had upgrading to ISUP Grade $\geq 2$. Thirteen percent of men had a suspicious DRE (done by their treating physician). Sensitivity, specificity, negative and positive predictive values of DRE to detect csPC were best with a PSA $<4 \mathrm{ng} / \mathrm{ml}(27 \%, 88 \%, 31 \%$, and $87 \%$, respectively). A suspicious DRE at CxPBx, particularly if the DRE at diagnosis was negative, was a 
predictor of csPC (odds ratio [OR] 2.34; $\mathrm{p}=0.038$ ). The main limitation of our study is the retrospective design and the lack of magnetic resonance imaging.

Conclusions: We believe DRE should still be used as part of AS and can predict the presence of csPC even with low PSA values. A suspicious nodule on DRE represents a higher risk of upgrading and should prompt further assessment.

\section{Introduction}

Active surveillance (AS) has become the standard of care for men with low risk prostate cancer (PC). ${ }^{1,2}$ One of the criteria used for including patients in AS protocols is the digital rectal examination (DRE). Most guidelines allow for cT2 patients to be offered AS, as long as the rest of the low risk criteria are fulfilled. . $^{3,4}$

AS follow up protocols vary between institutions. ${ }^{4-7}$ The follow up usually includes periodical prostate specific antigen (PSA) measurements, DRE and prostate biopsies $(\mathrm{PBx}){ }^{4}$ Most centers mandate a confirmatory PBx within 6-18 months from the initial diagnostic PBx, as the initial PBx can miss clinically significant prostate cancers (csPC) ${ }^{5}$

Large AS series have reported a consistent rate of pathological progression (upgrading and/or upstaging) at the confirmatory PBx of 19.9-28.1\%. ${ }^{5}$ Accurate and timely detection of patients with csPC who require treatment is crucial, although we also struggle to determine who may safely avoid or delay a confirmatory PBx. Risk factors for upstaging/upgrading include high volume ISUP Grade 1, suspicious lesions on magnetic resonance imaging (MRI), PSA density, race, and age. ${ }^{8-12}$

DRE is widely considered a component of clinical evaluation in men with prostate cancer diagnosis, although in AS the value of DRE is uncertain. Nonetheless, DRE involves mild discomfort and may require an additional patient visit. The objective of the present study was to analyse the value of DRE as a predictor of upgrading in a population of patients on AS.

\section{Methods}

After Research Ethics Board approval, we used the prospectively maintained PBx database from a large tertiary centre and retrieved patients treated with AS between 2006 and 2018. We included patients who were initially diagnosed with ISUP Grade 1 prostate cancer (in out center or somewhere else), where started on AS, and had at least one follow up PBx in our center. For patients who had more than 1 follow up PBx, we included only the first confirmatory $\mathrm{PBx}$ for the analysis, as the time to the following $\mathrm{PBx}$ is widely variable. As part of our AS protocol, all men initially managed with AS undergo a confirmatory PBx between 6-18 months from the diagnostic PBx. We also looked into patients who had both, 
diagnostic $\mathrm{PBx}$ and confirmatory $\mathrm{PBx}$ done in our center, of whom we had complete data to account for changes between the two time points. Data collected included age, number of $\mathrm{PBx}$, number of cores in the confirmatory PBx, PSA before the PBx, number of cores taken, prostate volume (PV), suspicious transrectal ultrasound results (TRUS), referral DRE (rDRE), DRE done by the radiologist at the time of the PBx (bxDRE), presence of PC (any) and presence of csPC (defined as ISUP Grade $\geq 2$ ). The rDRE was performed by the treating physician. The great majority of these DRE were done by Urologists (either UroOncologist or General Urologists), but some were done by Radiation Oncologists and a small proportion were performed by Family Physicians before the diagnostic PBx. These were done in clinic as part of the routine physical examination, both prior to the diagnostic $\mathrm{PBx}$ and during the AS before the confirmatory $\mathrm{PBx}$. Some men were referred to our center for the diagnostic PBx and then continued the AS by the group of Urologists in our center, and some were referred after the PC diagnosis was made with a biopsy done outside of our center. All of these men were being treated in our center after the diagnosis was made. We defined "suspicious DRE" as the presence of a palpable nodule compatible with prostate cancer, judged by the treating physician in clinic (rDRE) or the radiologist right before the biopsy (bxDRE). In our center we routinely do a systematic biopsy taking 12 cores (6 from each prostate lobule) for the first and subsequent PBx. Suspicious nodules on DRE or ultrasound are targeted obtaining extra cores (2-3 from each suspicious lesions), and in case of having a suspicious lesion on MRI we use a targeted fusion technique.

\section{Statistical analysis}

We did a descriptive analysis with median and interquartile ranges (IQR) for continuous variables, and proportions for discrete variables. We then calculated a Cohen kappa value between rDRE and bxDRE, as well as sensitivity, specificity and predictive values for the detection of csPC, stratified by PSA values (PSA $<4 \mathrm{ng} / \mathrm{ml}$, PSA $4-10 \mathrm{ng} / \mathrm{ml}$ and PSA $>10$ $\mathrm{ng} / \mathrm{ml})$.

Finally, we used univariable and multivariable logistic regression analysis to obtain the odds ratio (OR) of a positive rDRE to detect a csPC in the confirmatory PBx. rDRE was used for the inferential analyses, as it represent the current practice in which the initial or diagnostic DRE can be done by a variety of physicians with varying levels of expertise, as opposed to an physician who has done multiple biopsies and DREs, having constant feedback from pathological results for many years (bxDRE). The other predictors included into the models were chosen based on factors commonly used to guide decisions in patients on AS such as PSA, PV, number of cores taken at the biopsy, TRUS results and age. The multivariable model included all predictors that were statistically significant in the univariable analysis. 
We repeated the analyses using only the population of patients who had both diagnostic PBx and confirmatory PBx done in our center. In these group we analysed potential changes in PSA ("deltaPSA") and DRE from diagnostic PBx to confirmatory PBx. In this population we also categorized the changes in PSA, defined as:

- PSA decrease (PSAd) as a decrease in PSA from diagnostic PBx to confirmatory $\mathrm{PBx} \geq 2 \mathrm{ng} / \mathrm{ml}$

- PSA increase (PSAi) as an increase $\geq 2 \mathrm{ng} / \mathrm{ml}$

- Stable PSA (PSAs) if the PSA variability was less than $2 \mathrm{ng} / \mathrm{ml}$ from one biopsy to the other.

DRE dynamics were classified as:

- DRE-/- if DRE1 was non-suspicious at both diagnostic and confirmatory PBx

- DRE+/+ if DRE was suspicious at both diagnostic and confirmatory PBx

- DRE+/- if DRE was suspicious at diangositc PBx but non-suspicious at confirmatory PBx

- DRE-/+ if DRE was non-suspicious at diagnostic PBx and suspicious at confirmatory PBx.

All analyses were done using $\mathrm{R}$ version 3.4.3.

\section{Results}

A total of 19821 PBx were performed in our center between 2006 and 2018. Out of these, 2029 were first confirmatory PBx of unique patients initially diagnosed with low risk PC treated under AS. The population of patients with complete information on both, diagnostic PBx and confirmatory PBx in our center, consisted of 726 patients. Table 1 shows the patients and their associated PBx characteristics, showing an upgrading of $30.3 \%$ in all the confirmatory $\mathrm{PBx}$ and $21.9 \%$ in the cohort of patients who had both diagnostic and confirmatory $\mathrm{PBx}$ in our center.

Among all men with a confirmatory PBx $(n=2029)$, the rDRE was suspicious in $263(12.96 \%)$ and bxDRE in $463(22.82 \%)$. The agreement between rDRE and bxDRE was $82.8 \%$ with a Cohen kappa of 0.426 . Regarding the detection of csPC at confirmatory PBx, rDRE led to 497 false negative diagnoses (non-suspicious DRE with csPC at the confirmatory PBx) of which bxDRE detected 120 (24.1\%). Conversely, bxDRE led to 407 false negative diagnoses of which rDRE detected only 30 (7.4\%). For those with both diagnostic and confirmatory PBx, DRE agreement at diagnostic PBx, was $79.2 \%$, with a Cohen kappa of 0.451 , and at confirmatory PBx it was $82.2 \%$ with a Cohen kappa of 0.362 .

Table 2 shows the sensitivity, specificity, positive and negative predictive values of rDRE and bxDRE in all the confirmatory PBx $(n=2029)$ for the detection of csPC categorized by PSA values. We can see some differences between rDRE and bxDRE, mainly in terms of sensitivity. In Table 3 we present our inferential analysis for the 
prediction of Gleason upgrading in the whole cohort of men with confirmatory PBx. The predictors for upgrading were a suspicious rDRE, an elevated PSA, lower PV, a suspicious TRUS and older age.

For the 726 patients in whom we had both diagnostic and confirmatory $\mathrm{PBx}$, we found that most had DRE-/- (512, 70\%), 51 (7\%) had DRE+/+, $133(18 \%)$ had DRE+/-, and only $30(4 \%)$ had DRE-/+. We did a univariable and multivariable analysis including the variation of PSA (deltaPSA) and DRE (deltaDRE) in this subpopulation of patients, shown in Table 4. The predictors for upgrading were DRE -/+, an elevation in the PSA (deltaPSA), PV, older age and a suspicious TRUS.

Out of the men who had PSAd, PSAs and PSAi, 89 (18\%), $38(18 \%)$ and $68(29 \%)$ had csPC, respectively. As expected, PSAi (increase $\geq 2 \mathrm{ng} / \mathrm{ml}$ ) had the higher rate of Gleason upgrading, but PSAd and PSAs did not rule out the presence of csPC. Grouped by deltaDRE, of the men who had DRE-/-, DRE+/+, DRE+/- and DRE-/+, 104 (20\%), 15 (29\%), $29(22 \%)$ and $11(37 \%)$ had csPC, respectively.

\section{Discussion}

This study was conducted in a large population of AS patients undergoing their first confirmatory PBx. There was a slightly higher prevalence of suspicious DRE (13\%) compared to a screening population $(9.2 \%){ }^{13}$ This can be explained by the fact that these men are a selected population who already have a diagnosis of PC, although it seems to be a minor difference.

The rDRE has a low sensitivity (19\%) in the overall population, but a good specificity (90\%). This suggests that a suspicious rDRE can reliably signal presence of csPC indicating the need for a PBx or other studies (molecular biomarkers or MRI). Table 2 shows that sensitivity of rDRE for the detection of csPC in men undergoing a confirmatory PBx is higher with a lower PSA. Similarly, NPV is high, comparable to MRI, particularly with a PSA $<4 \mathrm{ng} / \mathrm{dl} .8,14,15$ The later could be in relation to a low prevalence of csPC in men with low PSA values. In contrast to reports in undiagnosed patients, ${ }^{16}$ in the AS population DRE was most useful in patients with a lower PSA. One explanation is that patients with a suspicious DRE and confirmed csPC at diagnostic PBx had already been selected out of the AS cohort (as a number of these suspicious DRE from the initial assessment have already been targeted in the diagnostic biopsy and represented csPC, that led to a treatment with curative intent as opposed to AS); thus a new nodule represents real disease progression. With these findings we can interpret that in case of finding a nodule on DRE we should do further testing (especially if this was not present in previous visits), but a normal or nonsuspicious DRE does not rule out the presence of csPC and the decision to pursue a new PBx should be based on other clinical factors (PSA dynamics, time from previous biopsy, tumor volume, MRI, etc.). In Table 2, we can observe a marked difference between rDRE 
and bxDRE, which may be a reflection of the experience or even the setting (the position of the patient, the constant review/feedback from pathological results, among others) in which physicians are doing biopsies in a regular basis. Such difference shows a clinical correlation, since bxDRE was suspicious in $24.1 \%$ of the csPC that were not detected on rDRE.

By looking into the population of whom we had information on both diagnostic and confirmatory $\mathrm{PBx}$, we were able to study the changes from one to another. As expected, those patients with an elevation of PSA $\geq 2 \mathrm{ng} / \mathrm{ml}$ had a higher frequency of csPC in the confirmatory PBx (29\%). The deltaPSA was a predictor of upgrading in both, univariable and multivariable analyses. This supports the current monitoring strategies. Regarding the DRE, Table 3 shows that in the case of not having information on the diagnostic PBx (all confirmatory PBx cohort), a suspicious DRE is a predictor of upgrading at confirmatory PBx. With more granularity, in the cohort of patients with information on both diagnostic and confirmatory PBx (Table 4), a "newly" suspicious DRE (DRE-/+) had a higher likelihood of upgrading, while the presence of a persistently suspicious DRE (DRE $+/+$ ) did not reach statistical significance. In the univariable and multivariable analysis only DRE-/+ was a predictor of the presence of csPC at confirmatory $\mathrm{PBx}$, despite modest numbers (30 patients, with 11 patients found with csPC at confirmatory $\mathrm{PBx}$ ). The lack of statistical significance of DRE+/+ as a predictor of upgrading may be related to the sample number (51 patients with 15 of them upgraded to csPC) but also to the fact that if they had a suspicious DRE since the beginning, it has likely been sampled in the diagnostic PBx and those with csPC have already been detected and treated accordingly.

We present evidence supporting the use of DRE as part of AS, adding information to decide whether to do a confirmatory PBx or not. Currently, even with the of new technology (MRI, biomarkers) available it is still recommended to always do at least one confirmatory PBx. ${ }^{17}$ Nonetheless, every information that could be obtained to predict the presence of a more aggressive cancer (csPC) can be used in clinic to decide upon performing further biopsies or even to risk stratify and consider a more conservative management for certain patients. We believe these results offer valuable evidence for the clinician, supporting further investigations in case of finding a positive DRE, regardless of the PSA dynamics. The need of a DRE in men with access to MRI has not been studied and could not be evaluated in the present study. The transformation from a non-suspicious DRE to a suspicious DRE should lead to a closer surveillance and throughout study in men on AS, and should be discussed with the patient.

The limitations of the study include the retrospective design and the missing information on the diagnostic $\mathrm{PBx}$, given that many of these were not done in our center. This design limited the available details on the DRE and had to be classified as suspicious vs. non-suspicious, as this is how it was recorded on the database. Similarly, since the 
rDRE was performed by a more heterogeneous group of physicians (particularly for the diagnostic PBx), rDRE had more interobserver variability compared to the bxDRE, which was performed by a limited number of physicians specialized in doing PBx. Likewise, the number of cores taken in each biopsy varied according to clinical criteria. Comparing the PSA changes over time or the PSA reported at diagnosis in the whole population would be useful and would allow better characterization of these variables, although we were able to do this in a significant number of patients $(n=726)$. Another limitation is the lack of MRI in this cohort. MRI has currently been incorporated into the follow up of AS men in many centers. ${ }^{17,18}$ In this cohort, MRI was not employed routinely. It is plausible that some cancers may be palpable without restricted diffusion, but this data is lacking. Further studies of the accuracy of DRE in the context of MRI are warranted. Also, the study was limited to the first follow up biopsy in an attempt to control for the time-to biopsy (time to subsequent $\mathrm{PBx}$ is much more variable after the first confirmatory biopsy), focusing the study on the initial period of AS.

\section{Conclusions}

Even though it cannot be used to exclude the presence of csPC, we believe DRE should still be used in the clinical evaluation of men being managed with AS, regardless of the PSA value. A suspicious nodule on DRE is associated with a higher risk of upgrading adding information that should be considered in the diagnostic workup and treatment decision. 


\section{References}

1. Chen RC, Rumble RB, Loblaw DA, et al. Active Surveillance for the Management of Localized Prostate Cancer (Cancer Care Ontario Guideline): American Society of Clinical Oncology Clinical Practice Guideline Endorsement. J Clin Oncol. 2016;34(18):2182-2190.

2. Morash C, Tey R, Agbassi C, et al. Active surveillance for the management of localized prostate cancer: Guideline recommendations. Can Urol Assoc J. 2015;9(56):171-178.

3. Briganti A, Fossati N, Catto JWF, et al. Active Surveillance for Low-risk Prostate Cancer: The European Association of Urology Position in 2018. Eur Urol. 2018;74(3):357-368.

4. Klotz L, Vesprini D, Sethukavalan P, et al. Long-term follow-up of a large active surveillance cohort of patients with prostate cancer. J Clin Oncol. 2015;33(3):272277.

5. Komisarenko M, Timilshina N, Richard PO, et al. Stricter Active Surveillance Criteria for Prostate Cancer do Not Result in Significantly Better Outcomes: A Comparison of Contemporary Protocols. J Urol. 2016;196(6):1645-1650.

6. Selvadurai ED, Singhera M, Thomas K, et al. Medium-term outcomes of active surveillance for localised prostate cancer. Eur Urol. 2013;64(6):981-987.

7. Tosoian JJ, Mamawala M, Epstein JI, et al. Intermediate and Longer-Term Outcomes From a Prospective Active-Surveillance Program for Favorable-Risk Prostate Cancer. J Clin Oncol. 2015;33(30):3379-3385.

8. Ahmed HU, El-Shater Bosaily A, Brown LC, et al. Diagnostic accuracy of multiparametric MRI and TRUS biopsy in prostate cancer (PROMIS): a paired validating confirmatory study. The Lancet. 2017;389(10071):815-822.

9. Fan Y, Zhai L, Meng Y, et al. Contemporary Epstein Criteria with Biopsy-Naive Multiparametric Magnetic Resonance Imaging to Prevent Incorrect Assignment to Active Surveillance in the PI-RADS Version 2.0 Era. Ann Surg Oncol. 2018;25(12):3510-3517.

10. Jain S, Loblaw A, Vesprini D, et al. Gleason Upgrading with Time in a Large Prostate Cancer Active Surveillance Cohort. J Urol. 2015;194(1):79-84.

11. Mamawala MM, Rao K, Landis P, et al. Risk prediction tool for grade reclassification in men with favourable-risk prostate cancer on active surveillance. BJU Int. 2017;120(1):25-31.

12. Radtke JP, Kuru TH, Bonekamp D, et al. Further reduction of disqualification rates by additional MRI-targeted biopsy with transperineal saturation biopsy compared with standard 12-core systematic biopsies for the selection of prostate cancer patients for active surveillance. Prostate Cancer Prostatic Dis. 2016;19(3):283-291.

13. Halpern JA, Shoag JE, Mittal S, et al. Prognostic Significance of Digital Rectal Examination and Prostate Specific Antigen in the Prostate, Lung, Colorectal and Ovarian (PLCO) Cancer Screening Arm. J Urol. 2017;197(2):363-368. 
14. Kasivisvanathan V, Rannikko AS, Borghi M, et al. MRI-Targeted or Standard Biopsy for Prostate-Cancer Diagnosis. N Engl J Med. 2018;378(19):1767-1777.

15. Moldovan PC, Van den Broeck T, Sylvester R, et al. What Is the Negative Predictive Value of Multiparametric Magnetic Resonance Imaging in Excluding Prostate Cancer at Biopsy? A Systematic Review and Meta-analysis from the European Association of Urology Prostate Cancer Guidelines Panel. Eur Urol. 2017;72(2):250-266.

16. Halpern JA, Oromendia C, Shoag JE, et al. Use of Digital Rectal Examination as an Adjunct to Prostate Specific Antigen in the Detection of Clinically Significant Prostate Cancer. J Urol. 2018;199(4):947-953.

17. Klotz L, Loblaw A, Sugar L, et al. Active Surveillance Magnetic Resonance Imaging Study (ASIST): Results of a Randomized Multicenter Prospective Trial. Eur Urol. 2019;75(2):300-309.

18. Schoots IG, Petrides N, Giganti F, et al. Magnetic resonance imaging in active surveillance of prostate cancer: a systematic review. Eur Urol. 2015;67(4):627-636.

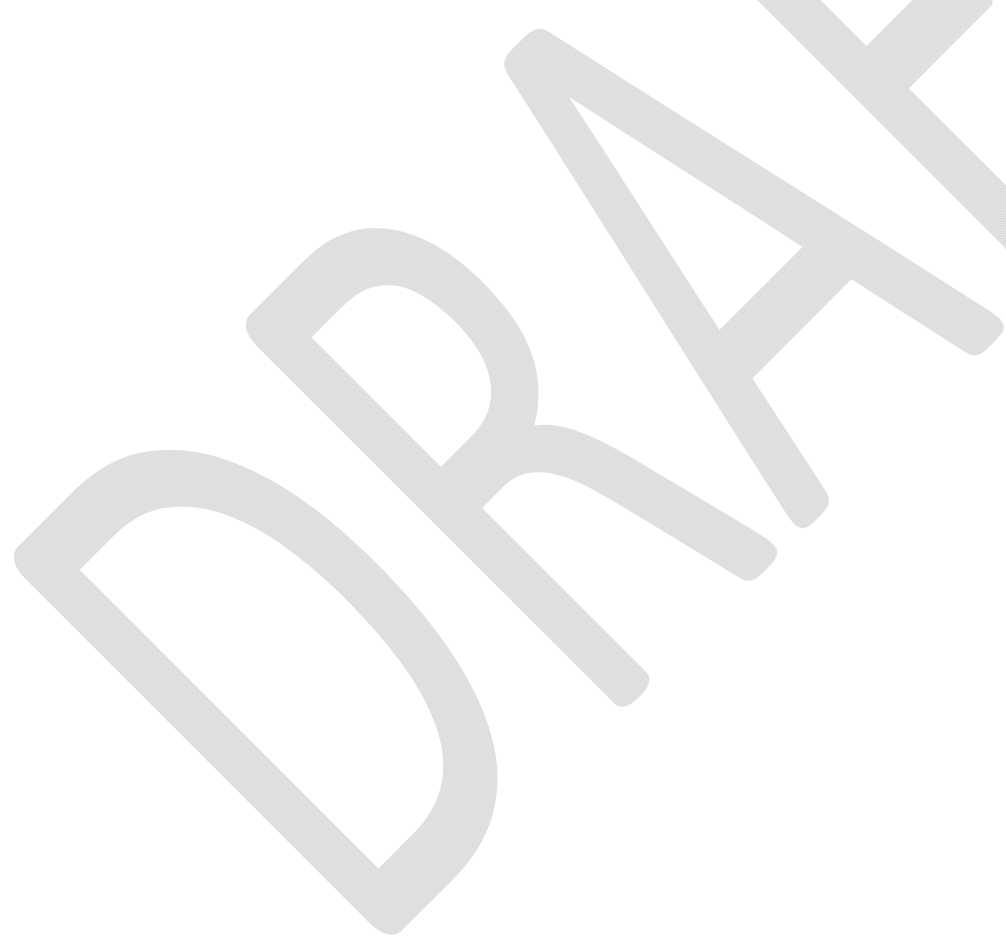




\begin{tabular}{|l|c|c|c|}
\hline \multicolumn{1}{|l|}{ Table 1. Patient and prostate biopsy (PBx) characteristics } \\
\hline & $\begin{array}{c}\text { All } \\
\text { confirmatory } \\
\text { PBx (n=2029) }\end{array}$ & $\begin{array}{c}\text { Diagnostic PBx } \\
\text { (n=726) }\end{array}$ & $\begin{array}{c}\text { Confirmatory PBx } \\
\text { in men with } \\
\text { diagnostic PBx } \\
\text { available (n=726) }\end{array}$ \\
\hline $\begin{array}{l}\text { Age in years, median } \\
\text { (IQR) }\end{array}$ & $65(60-71)$ & $63(58-68)$ & $65(60-70)$ \\
\hline $\begin{array}{l}\text { Number of cores, median } \\
(\text { IQR) }\end{array}$ & $16(14-17)$ & $12(10-12)$ & $16(15-17)$ \\
\hline $\begin{array}{l}\text { Prostate-specific antigen } \\
\text { in ng/ml, median (IQR) }\end{array}$ & $5.83(3.80-8.40)$ & $5.82(3.85-6.92)$ & $5.30(3.39-7.70)$ \\
\hline $\begin{array}{l}\text { Prostate volume in cc, } \\
\text { median (IQR) }\end{array}$ & $44(33-60)$ & $43(34-56)$ & $44(34-58)$ \\
\hline Suspicious TRUS, $n(\%)$ & $893(44 \%)$ & $232(32 \%)$ & $298(41 \%)$ \\
\hline Suspicious rDRE, $n(\%)$ & $263(12.96 \%)$ & $184(25.34 \%)$ & $81(11.16 \%)$ \\
\hline $\begin{array}{l}\text { Suspicious bxDRE, } n \\
(\%)\end{array}$ & $463(22.82 \%)$ & $185(25.48 \%)$ & $156(21.49 \%)$ \\
\hline Any cancer, $n(\%)$ & $1531(75.5 \%)$ & $726(100 \%)$ & $536(73.8 \%)$ \\
\hline $\begin{array}{l}\text { Upstaging to csPC, } n \\
(\%)\end{array}$ & $614(30.3 \%)$ & NA & $159(21.9 \%)$ \\
\hline
\end{tabular}

bxDRE: digital rectal examination done right before the biopsy by the person performing the biopsy; csPC: clinically significant prostate cancer (ISUP grade 2 or higher); IQR: interquartile ratio; PBx: prostate biopsy; rDRE: digital rectal examination done in clinic by the treating physician prior to the biopsy; TRUS: transrectal ultrasound.

Table 2. Sensitivity, specificity and positive and negative predictive values of rDRE and bxDRE in all the confirmatory biopsies $(n=2$ 029), stratified by PSA for the prediction of clinically significant prostate cancer

\begin{tabular}{|l|c|c|c|c|c|c|c|c|}
\hline & \multicolumn{4}{|c|}{ rDRE } & \multicolumn{4}{c|}{ bxDRE } \\
\hline & Overall & $\begin{array}{c}\text { PSA }<\mathbf{4} \\
\mathbf{n g} / \mathbf{m l}\end{array}$ & $\begin{array}{c}\text { PSA } \\
\mathbf{4 - 1 0} \\
\mathbf{n g} / \mathbf{m l}\end{array}$ & $\begin{array}{c}\text { PSA } \\
\mathbf{1 1 0} \\
\mathbf{n g} / \mathbf{m l}\end{array}$ & Overall & $\begin{array}{c}\text { PSA<4 } \\
\mathbf{n g} / \mathbf{m l}\end{array}$ & $\begin{array}{c}\text { PSA } \\
\mathbf{4}-\mathbf{1 0} \\
\mathbf{n g} / \mathbf{m l}\end{array}$ & $\begin{array}{c}\text { PSA } \\
>\mathbf{1 0} \\
\mathbf{n g} / \mathbf{m l}\end{array}$ \\
\hline Sensitivity & $19 \%$ & $27 \%$ & $18 \%$ & $17 \%$ & $34 \%$ & $35 \%$ & $32 \%$ & $37 \%$ \\
\hline Specificity & $90 \%$ & $88 \%$ & $91 \%$ & $85 \%$ & $82 \%$ & $80 \%$ & $83 \%$ & $79 \%$ \\
\hline PPV & $44 \%$ & $31 \%$ & $48 \%$ & $56 \%$ & $45 \%$ & $23 \%$ & $46 \%$ & $66 \%$ \\
\hline NPV & $72 \%$ & $87 \%$ & $71 \%$ & $49 \%$ & $74 \%$ & $87 \%$ & $73 \%$ & $54 \%$ \\
\hline
\end{tabular}

bxDRE: digital rectal examination done right before the biopsy by the person performing the biopsy; NPV: negative predictive value; PPV: positive predictive value; PSA: prostatespecific antigen; rDRE: digital rectal examination done in clinic by the treating physician prior to the biopsy. 


\begin{tabular}{|l|c|c|c|}
\hline $\begin{array}{l}\text { Table 3. Univariable and multivariable regression analyses predicting } \\
\text { upgrading in all confirmatory PBx (n=2029) }\end{array}$ \\
\hline & \multicolumn{2}{|c|}{ Clinically significant prostate cancer } \\
\cline { 2 - 4 } & OR & $\mathbf{9 5 \%}$ CI & p \\
\hline Univariable & & & $<0.001$ \\
\hline rDRE & 2.05 & $1.57-2.66$ & $<0.001$ \\
\hline PSA, ng/ml ${ }^{*}$ & 1.14 & $1.11-1.16$ & $<0.001$ \\
\hline PV, c & 0.99 & $0.98-0.99$ & 0.165 \\
\hline Number of cores taken & 1.03 & $0.99-1.06$ & $<0.001$ \\
\hline TRUS & 2.44 & $2.01-2.97$ & $<0.001$ \\
\hline Age, years & 1.06 & $1.05-1.08$ & \\
\hline Multivariable & & & 0.004 \\
\hline rDRE & 1.54 & $1.14-2.07$ & $<0.001$ \\
\hline PSA (ng/ml) & 1.15 & $1.12-1.18$ & $<0.001$ \\
\hline PV, cc & 0.98 & $0.97-0.98$ & $<0.001$ \\
\hline Suspicious TRUS & 2.05 & $1.66-2.53$ & $<0.001$ \\
\hline Age (year) & 1.06 & $1.04-1.07$ & \\
\hline
\end{tabular}

*The OR was calculated using PSA as a continuous variable, representing each $1.0 \mathrm{ng} / \mathrm{ml}$ increase. CI: confidence intervals; OR: odds ratio; PBx: prostate biopy; PSA: prostatespecific antigen; PV: prostate volume; rDRE: digital rectal examination done in clinic by the treating physician prior to the biopsy; TRUS: transrectal ultrasound. 


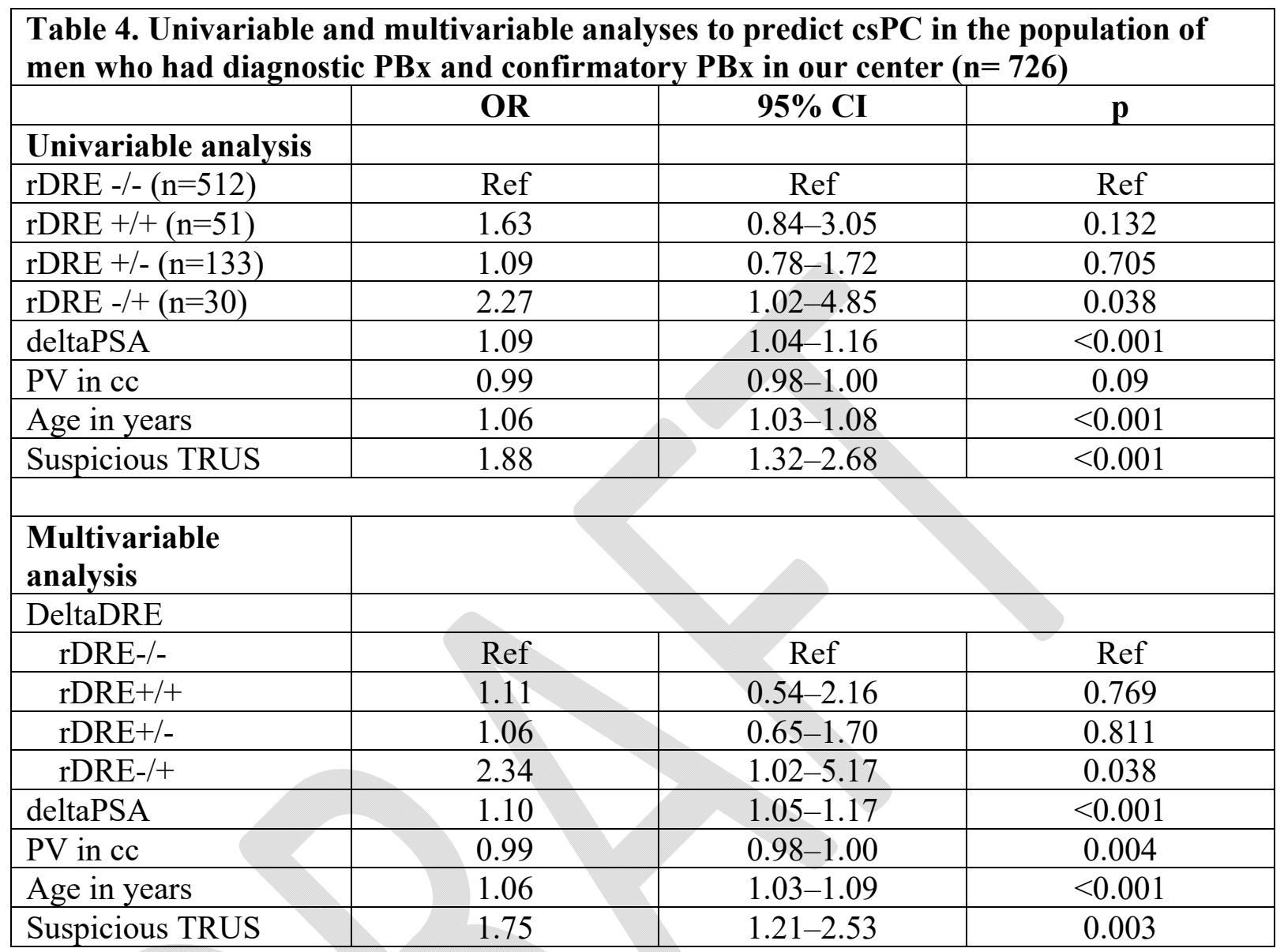

CI: confidence intervals; csPC: clinically significant prostate cancer (ISUP grade 2 or higher); deltaPSA: variation of prostate-specific antigen from the diagnostic prostate biopsy to the confirmatory prostate biopsy; OR: odds ratio; PBx: prostate biopsy; PV: prostate volume; rDRE: digital rectal examination done in clinic by the treating physician prior to the biopsy; TRUS, transrectal ultrasound. 